\title{
An Application of ARQ Optimization Algorithm
}

\author{
Eduardo Rodrigues Vale, Julio Cesar R. Dal Bello \\ ${ }^{1,2}$ (Telecommunications Department, School of Engineering / Federal Fluminense University, Brazil)
}

\begin{abstract}
In a previous work [1], we presented a development with the objective of defining an algorithm able to minimize the extra amount of power utilized in the transmission of information from mobile devices in the uplink direction, relatively to the use of the technique called ARQ (Automatic Repeat Request). Although the above algorithm had been designed for use in HSPA networks, it finds application in other systems employing the $A R Q$ technology. Here we will be analyzing an application of the algorithm, with the consequent analysis of the results obtained.
\end{abstract}

Keywords: ARQ, UMTS, BER, HSPA, LTE.

\section{INTRODUCTION}

The main objective of this work is to test a methodology developed in a previous work [1] aiming ARQ optimization, relatively to the mean power required to transfer the information from the origin to the destination, accordingly to a given error probability.

The ARQ technology proves to be very useful for data transmission in WCDMA systems. Because this process is executed at RLC (Radio Link Control) level [2], the resultant delay does not permit its utilization for real time services [3]. This situation has changed with the introduction of HSPA systems, where some ARQ functionalities were transfered to the Physical level (PHY) [4, 5]. The main consequence was a delay reduction in the information transfer, thus allowing the ARQ utilization also for real time services. This is also the case of the LTE (Long Term Evolution) systems of the Fourth Generation.

The paper is organized as follows: after this Introduction, Section II describes the application of the theory described in Reference [1] and Section III presents the related conclusions.

\section{THEORY APPLICATION}

Considering the results of the theory showed in Reference [1], it will be presented here an application of the developed algorithm, and described in detail in the cited reference. All the computations that follows were developed in Matlab (a product from Mathworks).

It is considered the development of an ARQ process with $n=2,3$ and 4 stages (i.e. two, three and four repetitions) for an UMTS system. In this example, the turbo code is receiving frames of $\mathrm{N}=600$ bits, and the interval of interest for Eb/No is defined from $-4 \mathrm{~dB}$ to $-6 \mathrm{~dB}$. The Tables 1, 2 and 3 show the results obtained for $n=2,3$ and 4 , respectively. The first column of these tables presents the $P($ error $)(\mathrm{dB})$ variation and the subsequent colums show the corresponding $x_{n}$ values (also in $\mathrm{dB}$ ) that minimizes $C_{m}$, indicated next to the last column. Finnaly, the last column presents the mean delay time for each case.

Table $1-N=600$ and $n=2$ case

\begin{tabular}{|c|c|c|c|c|c|}
\hline $\begin{array}{c}P(\text { error }) \\
(\mathrm{dB})\end{array}$ & $x_{1}$ & $x_{2}$ & $x_{3}$ & $C_{m}$ & $\begin{array}{c}\text { Delay } \\
(\mathrm{dB})\end{array}$ \\
\hline-4.0 & -4.9000 & -4.8000 & 0.8884 & -2.1546 & 1.4107 \\
\hline-4.2 & -4.9000 & -4.8000 & 0.9574 & -2.1411 & 1.4107 \\
\hline-4.4 & -4.9000 & -4.8000 & 1.0243 & -2.1279 & 1.4107 \\
\hline-4.6 & -4.9000 & -4.8000 & 1.0896 & -2.1148 & 1.4107 \\
\hline-4.8 & -4.9000 & -4.8000 & 1.1536 & -2.1019 & 1.4107 \\
\hline-5.0 & -4.9000 & -4.8000 & 1.2166 & -2.0890 & 1.4107 \\
\hline-5.2 & -4.9000 & -4.8000 & 1.2788 & -2.0761 & 1.4107 \\
\hline-5.4 & -4.9000 & -4.8000 & 1.3407 & -2.0631 & 1.4107 \\
\hline-5.6 & -4.9000 & -4.8000 & 1.4025 & -2.0500 & 1.4107 \\
\hline-5.8 & -4.9000 & -4.8000 & 1.4645 & -2.0367 & 1.4107 \\
\hline
\end{tabular}


Table $2-N=600$ and $n=3$ case

\begin{tabular}{|c|c|c|c|c|c|c|}
\hline $\begin{array}{c}P(\text { error }) \\
(\mathrm{dB})\end{array}$ & $\begin{array}{c}x_{1} \\
(\mathrm{~dB})\end{array}$ & $\begin{array}{c}x_{2} \\
(\mathrm{~dB})\end{array}$ & $\begin{array}{c}x_{3} \\
(\mathrm{~dB})\end{array}$ & $\begin{array}{c}x_{4} \\
(\mathrm{~dB})\end{array}$ & $\begin{array}{c}C_{m} \\
(\mathrm{~dB})\end{array}$ & $\begin{array}{c}\text { Delay } \\
(\mathrm{sec})\end{array}$ \\
\hline-4.0 & -4.9000 & -4.8000 & -4.8000 & 0.6985 & -2.3185 & 1.4402 \\
\hline-4.2 & -4.9000 & -4.8000 & -4.8000 & 0.7750 & -2.3139 & 1.4402 \\
\hline-4.4 & -4.9000 & -4.8000 & -4.8000 & 0.8481 & -2.3095 & 1.4402 \\
\hline-4.6 & -4.9000 & -4.8000 & -4.8000 & 0.9184 & -2.3052 & 1.4402 \\
\hline-4.8 & -4.9000 & -4.8000 & -4.8000 & 0.9864 & -2.3009 & 1.4402 \\
\hline-5.0 & -4.9000 & -4.8000 & -4.8000 & 1.0526 & -2.2967 & 1.4402 \\
\hline-5.2 & -4.9000 & -4.8000 & -4.8000 & 1.1173 & -2.2926 & 1.4402 \\
\hline-5.4 & -4.9000 & -4.8000 & -4.8000 & 1.1808 & -2.2884 & 1.4402 \\
\hline-5.6 & -4.9000 & -4.8000 & -4.8000 & 1.2434 & -2.2843 & 1.4402 \\
\hline-5.8 & -4.9000 & -4.8000 & -4.8000 & 1.3055 & -2.2801 & 1.4402 \\
\hline-6.0 & -4.9000 & -4.8000 & -4.8000 & 1.3673 & -2.2759 & 1.4402 \\
\hline
\end{tabular}

Table $3-N=600$ and $n=4$ case

The Figure 1 shows the $C_{m}$ values for the cases presented in the previous tables. Here it is also included, for comparison purposes, the situation with no repetition $(n=1)$. It is interesting to observe that it is not feasible to increase the number of repetitions beyond $n=3$ or 4 , because this does not contribute to an appreciate decrease in $C_{m}$ and also gives an increase in the delay values.

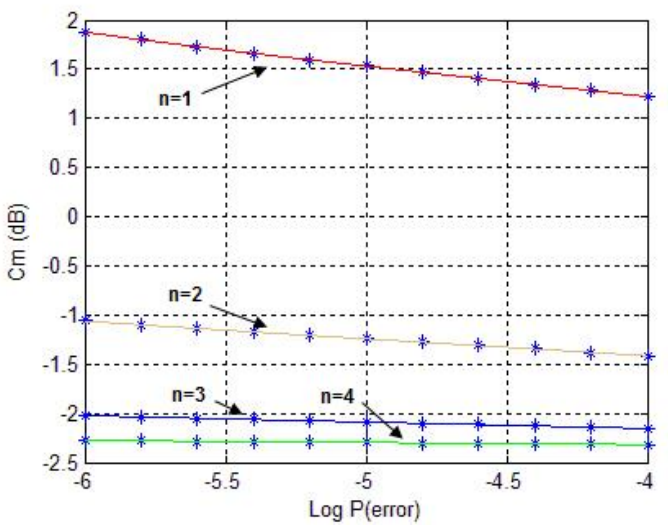

Figure $1-C_{m} \times \log P($ error $)$ values for $n=1,2,3$ and 4

Another situation of interest is the minimization of the maximum $x_{n}$ value for a given process, instead of $C_{m}$. In this case, for this calculation it is necessary only to change the step 8 of the algorithm of section 4 of Reference [1] to:

8. Select the least of the maximum $x_{n}$ values for each $P($ error $)$ in a given process, obtained from all the above interactions.

The Tables 4 and 5 present the results for this situation.

\begin{tabular}{|c|c|c|c|c|}
\hline $\begin{array}{c}P \text { (error) } \\
(\mathrm{dB})\end{array}$ & $\begin{array}{c}x_{1} \\
(\mathrm{~dB})\end{array}$ & $\begin{array}{c}x_{2} \\
(\mathrm{~dB})\end{array}$ & $\begin{array}{c}C_{m} \\
(\mathrm{~dB})\end{array}$ & $\begin{array}{c}\text { Delay } \\
(\mathrm{sec})\end{array}$ \\
\hline-4.0 & 0.5000 & 0.5016 & 0.5434 & 1.0100 \\
\hline-4.2 & 0.5000 & 0.5894 & 0.5443 & 1.0100 \\
\hline-4.4 & 0.5000 & 0.6717 & 0.5451 & 1.0100 \\
\hline-4.6 & 0.6000 & 0.6601 & 0.6260 & 1.0059 \\
\hline-4.8 & 0.6000 & 0.7385 & 0.6265 & 1.0059 \\
\hline-5.0 & 0.7000 & 0.7206 & 0.7146 & 1.0034 \\
\hline-5.2 & 0.7000 & 0.7961 & 0.7149 & 1.0034 \\
\hline-5.4 & 0.7000 & 0.8683 & 0.7151 & 1.0034 \\
\hline-5.6 & 0.8000 & 0.8456 & 0.8080 & 1.0018 \\
\hline-5.8 & 0.8000 & 0.9159 & 0.8082 & 1.0018 \\
\hline-6.0 & 0.8000 & 0.9841 & 0.8083 & 1.0018 \\
\hline
\end{tabular}


Table $4-N=600$ and $n=2$ case. Minimization of $x_{n}$

\begin{tabular}{|c|c|c|c|c|c|}
\hline $\begin{array}{c}P \text { (error) } \\
(\mathrm{dB})\end{array}$ & $\begin{array}{c}x_{1} \\
(\mathrm{~dB})\end{array}$ & $\begin{array}{c}x_{2} \\
(\mathrm{~dB})\end{array}$ & $\begin{array}{c}x_{3} \\
(\mathrm{~dB})\end{array}$ & $\begin{array}{c}C_{m} \\
(\mathrm{~dB})\end{array}$ & $\begin{array}{c}\text { Delay } \\
(\mathrm{sec})\end{array}$ \\
\hline-4.0 & 0.0000 & 0.2000 & 0.2129 & 0.3563 & 1.0762 \\
\hline-4.2 & 0.1000 & 0.2000 & 0.2487 & 0.3577 & 1.0557 \\
\hline-4.4 & 0.2000 & 0.2000 & 0.2750 & 0.3784 & 1.0391 \\
\hline-4.6 & 0.1000 & 0.3000 & 0.3724 & 0.3550 & 1.0551 \\
\hline-4.8 & 0.3000 & 0.3000 & 0.3054 & 0.4167 & 1.0260 \\
\hline-5.0 & 0.3000 & 0.3000 & 0.4092 & 0.4168 & 1.0260 \\
\hline-5.2 & 0.3000 & 0.4000 & 0.4134 & 0.4164 & 1.0257 \\
\hline-5.4 & 0.4000 & 0.4000 & 0.4177 & 0.4736 & 1.0166 \\
\hline-5.6 & 0.3000 & 0.5000 & 0.5032 & 0.4169 & 1.0256 \\
\hline-5.8 & 0.4000 & 0.5000 & 0.5070 & 0.4739 & 1.0165 \\
\hline-6.0 & 0.5000 & 0.5000 & 0.5025 & 0.5447 & 1.0101 \\
\hline
\end{tabular}

Table $5-N=600$ and $n=3$ case. Minimization of $x_{n}$

The Figure 2 shows the variations of $C_{m}(d B)$ x $\log P($ error $)$ for the situations of Tables 4 and 5 and the case of $n=1$ for comparison purposes.

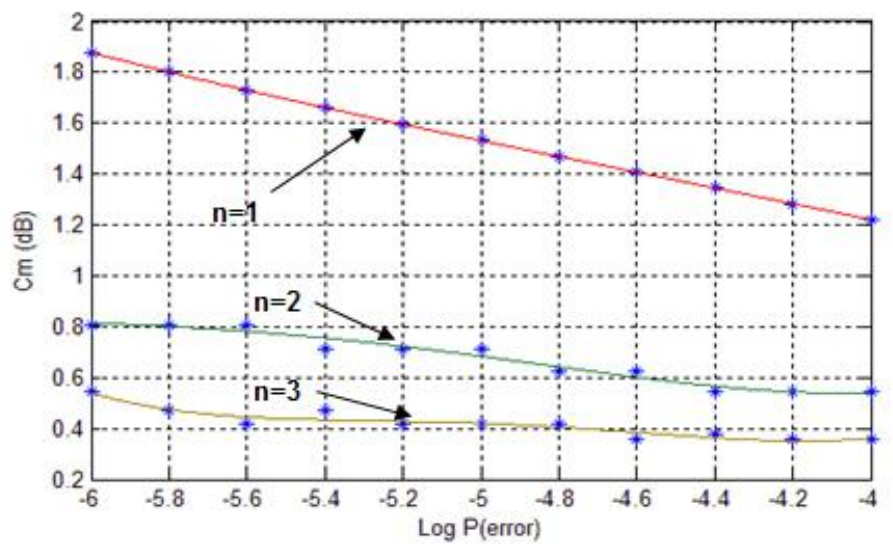

Figure $2-C_{m} \mathrm{x} \log P($ error $)$ values for $n=1,2$, and 3. Minimization of $x_{n}$

Comparing the results presented by Figures 1 and 2 gives the conclusion that, as expected, the corresponding $C_{m}$ values for the case of "Minimization of $x_{n}$ " are superior than those obtained for the previous situation of " $C_{m}$ Minimization", but the maximum $x_{n}$ values are lower than the corresponding ones for the " $C_{m}$ Minimization" case, as can be seen by comparison of the previous Tables. Also, the "Minimization of $x_{n}$ " case gives lower mean delay values, as stated by the last column of the corresponding Tables.

\section{Conclusions}

The ARQ process is a powerful technique used in UMTS systems, with the main objective to provide the information transfer with a certain degree of error rate. In the case of UMTS systems, it is a task to transfer the information packets in the air interface using the minimum possible amount of power, while maintaining the QoS of the service. This is particularly important in the uplink direction of transmission, because the saving of power transmission represents more economy of cellular batteries and reduced interference levels in the Node B station. This last effect is important for decreasing de noise rise levels, thus allowing more active users per cell.

\section{References}

[1] Vale, E. R. and Dal Bello, J. C. R., ARQ Optimization for UMTS Networks, IOSR Journals, may 2014.

[2] 3GPP Document, Radio Link Control (RLC) Protocol Specification, TS 25.322, v6.0.0, December 2012.

[3] Hammer F., Reichl P., Nordstrom T., Kubin G., Corrupted Speech Data Considered Useful, Acta Acustica, Vol.90, No. 6, pp 10521060, Nov/Dec 2004.

[4] Aftelak S. and Bhatoolaul, Rate Matching Attribute Settings and Error Rate Performance Sensitivity for Selected UMTS FDD Services, IEEE Semiannual Vehicular Technology Conference Final Program, October 6-9, 2003.

[5] Vale E. R., Brandão J.C., Grivet M., UMTS Rate Matching Optimization, International Association of Science and Technology for Development - IASTED, CSA 2005, Banff, Canada, July 2005. 